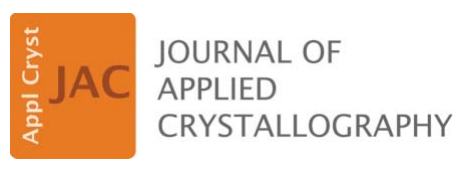

ISSN 1600-5767

Received 11 October 2019

Accepted 7 January 2020

Edited by F. Meilleur, Oak Ridge National Laboratory, USA, and North Carolina State University, USA

Keywords: neutron imaging; wavelengthresolved neutron tomography; Bragg-edge imaging.

Supporting information: this article has supporting information at journals.iucr.org/j

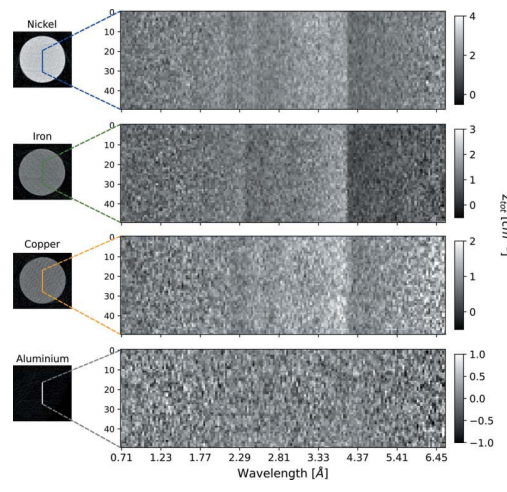

(C) 2020 International Union of Crystallography

\section{Bragg-edge attenuation spectra at voxel level from 4D wavelength-resolved neutron tomography}

\author{
Chiara Carminati, ${ }^{a *}$ Markus Strobl, ${ }^{a, b}$ Triestino Minniti, ${ }^{c}$ Pierre Boillat, ${ }^{a, d}$ Jan \\ Hovind, ${ }^{a}$ Manuel Morgano, ${ }^{a}$ Thomas Holm Rod, ${ }^{\text {e }}$ Efthymios Polatidis, ${ }^{a}$ Jacopo \\ Valsecchi, ${ }^{a, f}$ David Mannes, ${ }^{a}$ Winfried Kockelmann ${ }^{f}$ and Anders Kaestner ${ }^{a}$
}

\footnotetext{
aLaboratory for Neutron Scattering and Imaging, Paul Scherrer Institut, Villigen, Switzerland, ${ }^{\mathbf{b}}$ Niels Bohr Institute, University of Copenhagen, Nørregade 10, 1165 Copenhagen, Denmark, ${ }^{\mathbf{C}}$ UKAEA - United Kingdom Atomic Energy Authority, Culham Science Centre, Abingdon OX14 3DB, UK, ${ }^{\mathbf{d} E l e c t r o c h e m i s t r y ~ L a b o r a t o r y, ~ P a u l ~ S c h e r r e r ~ I n s t i t u t, ~}$ Villigen, Switzerland, ${ }^{\mathbf{e} E u r o p e a n}$ Spallation Source ERIC, Sweden, and ${ }^{\mathbf{f}}$ STFC - Rutherford Appleton Laboratory, ISIS Facility, Harwell, Oxfordshire, UK. *Correspondence e-mail: chiara.carminati@psi.ch
}

4D wavelength-resolved neutron tomography of a reference sample made of several polycrystalline materials, namely nickel, iron, titanium, lead, copper and aluminium, is presented. Data were acquired using the time-of-flight transmission imaging method at the IMAT beamline at the ISIS pulsed neutron source. Wavelength-dispersive tomography reconstruction was computed using filtered back projection, allowing wavelength-resolved total-cross-section retrieval for each voxel in the reconstructed volume of the sample. The need for background correction to enable quantitative results and analysis is discussed, and the achieved 3D spatial resolution with respect to the obtained Bragg-edge pattern quality is investigated.

\section{Introduction}

Bragg-edge neutron transmission is emerging as a powerful technique to study in a nondestructive way the microstructural information of crystalline materials (Santisteban et al., 2002a; Woracek et al., 2018), such as phase (Steuwer et al., 2004, 2005; Woracek et al., 2014; Makowska et al., 2015, 2016, 2017; Vitucci et al., 2018), texture (Santisteban et al., 2006, 2012) and stress state (Santisteban et al., 2001, 2002b; Steuwer et al., 2001, 2003; Abbey et al., 2009; Woracek et al., 2011; Strobl et al., 2012; Iwase et al., 2012; Wensrich et al., 2016a,b; Hendriks et al., 2017; Brooks et al., 2018; Ramadhan et al., 2019). With the time-offlight (TOF) method, it is possible to compute the spatially resolved wavelength-dependent transmission and in principle the total cross section of a sample (Kockelmann et al., 2007; Lehmann et al., 2009) for each pixel position of a TOF imaging detector (Tremsin et al., 2015).

Transmission spectra of polycrystalline materials, such as most metals, exhibit in the thermal and cold neutron energies specific patterns characterized by well defined and sudden increases of transmitted intensity (Bragg edges), due to coherent elastic scattering of neutrons with wavelength $\lambda$ at specific $h k l$ lattice planes with inter-planar distance $d$. According to Bragg's law, $\lambda=2 d_{h k l} \sin \theta$, the Bragg edges occur when $\lambda=2 d_{h k l}$, that is when the scattering angle $(2 \theta)$ is equal to $180^{\circ}$, because beyond that wavelength no further Bragg scattering can take place at these specific lattice planes. Therefore, analysis of the shape and position of the Bragg edges allows quantitative characterization of the crystallographic properties of a specimen (Sato, 2017; Song et al., 2017; Woracek et al., 2018; Minniti, 2019). 
Wavelength-selective neutron tomography can enhance the contrast between different materials or phases of the same material, for example, by selecting a specific range or a limited set of a few different wavelengths from the incident neutron spectrum (Kardjilov et al., 2003, 2004; Treimer et al., 2006; Woracek et al., 2014; Makowska et al., 2015, 2017). With the option of wavelength-resolved tomographic scanning, some crystallographic properties of a studied material (e.g. lattice spacing, phase) can be mapped in three dimensions. The straightforward benefit is the possibility to study how these properties vary throughout the sample volume. Assuming an isotropic orientation distribution along the beam direction of the crystal grains in a polycrystalline material, the filtered back projection algorithm may be applied for tomographic reconstruction (Woracek et al., 2014). In the case of single crystals (Venkatakrishnan et al., 2019) or strain tomography (Abbey et al., 2009; Wensrich et al., 2016b; Hendriks et al., 2017), where the anisotropy of the probed characteristics has to be considered, iterative techniques with specific boundary conditions have been implemented to address the tomographic reconstruction.

We present in this paper a TOF wavelength-dispersive neutron tomography study of a reference sample made of several metallic components, namely aluminium, iron, copper, lead, nickel and titanium. The sample was originally designed for contrast analysis in white-beam neutron tomography, as a round-robin facility reference (Kaestner et al., 2013). This study already indicated that there are differences in attenuation coefficients depending on the neutron spectrum. The aim of the current work is to study this sample through wavelength-dispersive tomography, where full attenuation spectra are computed for all voxels of the $3 \mathrm{D}$ reconstruction of the sample. The total cross sections for isotropic powder-like materials are considered as reference and used for comparison with the computed spectra of the studied materials.

The ability to reconstruct full Bragg-edge spectra in three dimensions at a voxel-by-voxel level has a distinct advantage over previous tomography approaches using diffraction contrast, namely that the investigated parameters are not fixed beforehand. Hence, the results allow for considering and investigating different features, such as phase fractions for a number of different phases, without the need for prior knowledge of the content of the sample. In addition, the approach enables straightforward identification of biases of, for example, texture related to phase measurements, which would invalidate conventional reconstructions (Woracek et al., 2014) without becoming apparent.

\section{Experiment}

\subsection{Sample description}

The sample was produced at Paul Scherrer Institut (PSI) in the framework of an international collaboration through the International Atomic Energy Agency (IAEA) and consists of a cylinder (30 mm diameter, $30 \mathrm{~mm}$ height) made of aluminium as matrix material. On a concentric ring of $18 \mathrm{~mm}$ diameter within this cylindrical Al matrix, six cylindrical insets with diameters of $6 \mathrm{~mm}$ are arranged, each made of a different material: lead, copper, nickel, iron, titanium and aluminium itself, respectively (Fig. 1). The sample was originally meant as reference object for quantification of the linear attenuation coefficients and characterization of corresponding contrast in white-beam neutron tomography. Kaestner et al. (2013) presented the round-robin test results for the case of the ICON (Kaestner et al., 2011) and NEUTRA (Lehmann et al., 2001) beamlines at PSI.

\subsection{TOF tomography}

The TOF tomography experiment was carried out at the IMAT beamline at the ISIS pulsed spallation source of the Rutherford Appleton Laboratory in the UK (Minniti et al., 2018; Ramadhan et al., 2019). TOF radiographs were taken for 251 projections uniformly distributed over a $360^{\circ}$ tomographic range. The exposure time for each TOF imaging projection was $10 \mathrm{~min}$. A microchannel plate/Timepix (MCP) detector was used for TOF imaging. The detector features $2 \times 2$ arrays of chips, resulting in a $512 \times 512$ pixel matrix with $55 \mu \mathrm{m}$ pixel size (Tremsin et al., 2015). For each projection, 2970 images corresponding to the individual TOF bins were obtained in three acquisition windows per period, accumulated over the exposure time but separated by read out gaps. Given the flight path $(L)$ of about $56 \mathrm{~m}$, TOF to wavelength conversion was computed according to $\lambda=\hbar t /(m L)$, where $t$ is the TOF, $\hbar$ is the reduced Planck constant and $m$ is the neutron mass. The resulting spectral range spanned from 0.7 to $6.7 \AA$, and the raw data sets had an original wavelength bin width of $0.00144 \AA$ for the first two acquisition windows $(\lambda<3.37 \AA)$ and $0.00288 \AA$ for the third window $(\lambda>3.38 \AA)$. The narrower bin
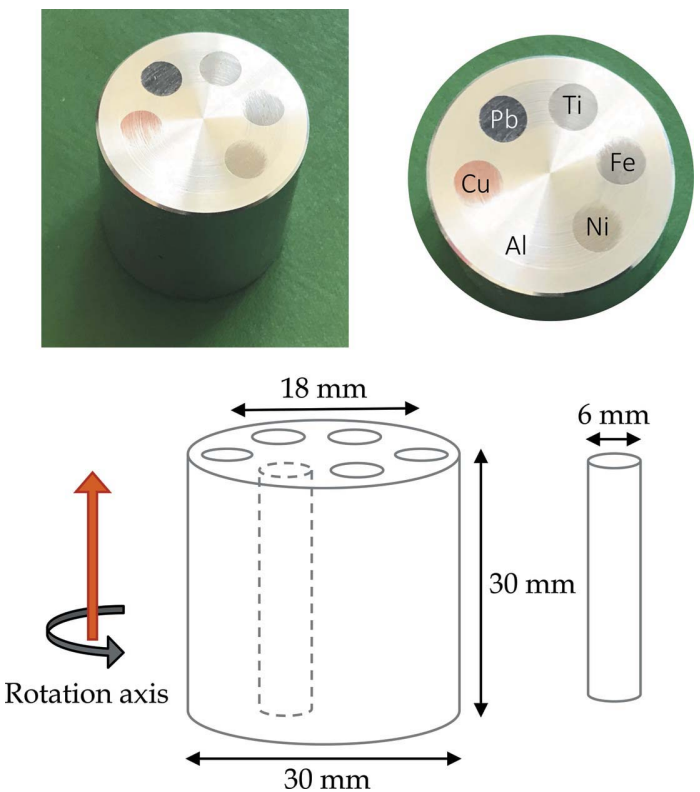

Figure 1

$30 \mathrm{~mm}$

The reference sample. A cylinder made of Al contains insets comprising different metals $(\mathrm{Pb}, \mathrm{Cu}, \mathrm{Ni}, \mathrm{Fe}, \mathrm{Ti}$ and $\mathrm{Al})$ arranged on a concentric ring. The sample was rotated around its longitudinal axis during the tomographic scan. 
width at shorter wavelengths is applied because of the smaller pulse burst widths for short-wavelength neutrons (Ramadhan et al., 2019). In addition to the tomographic scan, three sets of TOF open-beam measurements using the same acquisition settings were taken for image normalization. Overall, the experiment time to obtain all data was about $43 \mathrm{~h}$.

\section{Data treatment}

\subsection{Binning}

Detector event overlap correction for all acquisition windows was performed as described by Tremsin et al. (2014). Rebinning along the wavelength axis was achieved by summing stacks of 18 consecutive images, resulting in spectra with 165 bins for each projection. This choice was made on the one hand to still match the intrinsic instrumental wavelength resolution $(\Delta \lambda / \lambda<0.9 \%$ at $\lambda \sim 2.6 \AA$; Ramadhan et al., 2019), while increasing on the other hand the counting statistics of each bin to provide tomographic reconstructions with sufficiently high signal-to-noise ratio (SNR) for the quantitative analysis. The resulting wavelength increment after binning was $0.02592 \AA$ for the first two windows and $0.05184 \AA$ for the third window.

\subsection{Tomographic reconstruction}

Tomographic reconstruction was performed using the custom-made in-house software MuhRec (Kaestner, 2011; Kaestner \& Carminati, 2019), an open-source and multiplatform tool developed at PSI for neutron and X-ray tomography reconstruction. In a first step a white-beam tomogram was computed by summing all wavelength bins for each projection. The purpose of the white-beam reconstruction is to tune the reconstruction parameters (geometry and artifact reduction), profiting from a higher SNR. Flat-field normalization was applied as the first operation in the processing chain, followed by spot correction and filtered back projection.
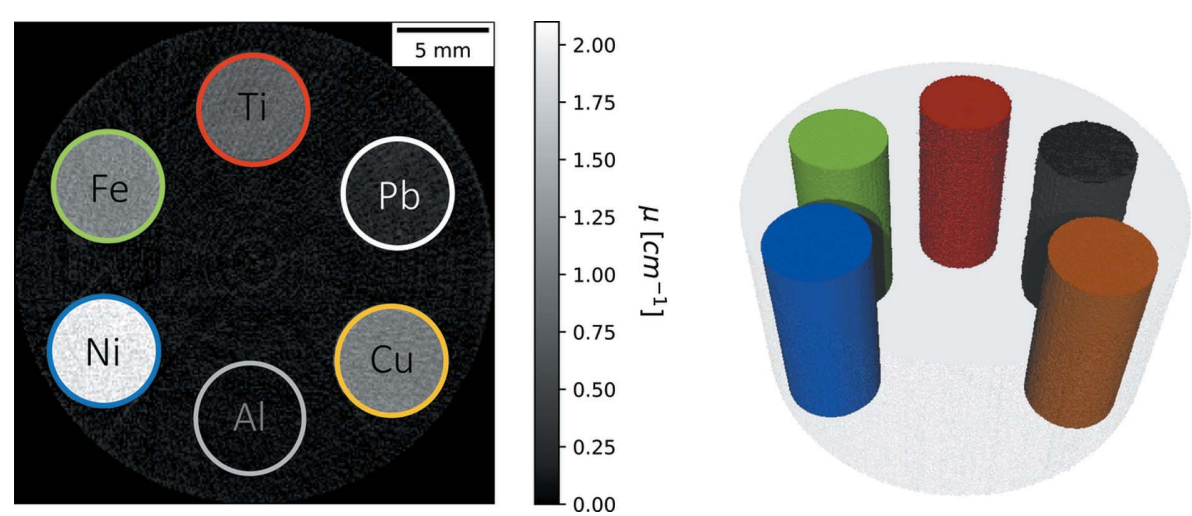

Figure 2

Results for the white-beam tomographic reconstruction. On the left, a cross section taken in the middle of the sample is shown, where gray values correspond to the linear attenuation coefficients $(\mu$ $\left[\mathrm{cm}^{-1}\right]$ ); on the right, a color-coded 3D rendering of the reconstructed volume, computed with VGStudio MAX (Volume Graphics GmbH, Heidelberg, Germany). The segmentation of individual insets was made on the basis of the attenuation coefficients of the white-beam tomography.
The wavelength-dispersive tomographic reconstruction using the 165 wavelength bins of tomographic projection sets was computed by invoking the command line interface of $M u h R e c$ from a loop in a processing script. The entire process was fully automated and required $2.5 \mathrm{~h}$ of computation time on a standard workstation (Xeon w-2125, 8 cores, $4.6 \mathrm{GHz}$, $32 \mathrm{~Gb}$ RAM). For both white-beam and wavelength-resolved tomography, the effect of additive background was estimated empirically and subtracted from the raw data. A detailed explanation of the background correction is presented in Section 4.

\subsection{Bragg-edge fitting}

Bragg-edge fitting was performed on the resulting local spectra in the reconstructed volume by the nonlinear fitting of an analytical model of the edge function, in order to assess the quality and potential for quantitative analyses.

According to Santisteban et al. (2001), the normalized transmitted intensity can be expressed as a function of $\lambda$ :

$$
\begin{aligned}
\frac{I_{\mathrm{t}}(\lambda)}{I_{0}(\lambda)}= & \exp \left[-\left(a_{0}+b_{0} \lambda\right)\right]\left(\exp \left[-\left(a_{h k l}+b_{h k l} \lambda\right)\right]\right. \\
& \left.+\left\{1-\exp \left[-\left(a_{h k l}+b_{h k l} \lambda\right)\right]\right\} B(\lambda)\right),
\end{aligned}
$$

where $I_{\mathrm{t}}(\lambda)$ and $I_{0}(\lambda)$ are the transmitted and incident wavelength-dependent intensities, respectively. The two exponential functions defined by the parameters $a_{0}, b_{0}$ and $a_{h k l}, b_{h k l}$ describe the transmitted intensity after and before the Bragg edge, modeled by the line shape $B$. The $B$ function consists of a smoothed switch approximating the Bragg-edge line shape, described by the Kropff model (Kropff et al., 1982) as

$$
\begin{aligned}
B(\lambda)= & \frac{1}{2}\left[\operatorname{erfc}\left(-\frac{\lambda-\lambda_{h k l}}{2^{1 / 2} \sigma}\right)-\exp \left(-\frac{\lambda-\lambda_{h k l}}{\tau}+\frac{\sigma^{2}}{2 \tau^{2}}\right)\right. \\
& \left.\times \operatorname{erfc}\left(-\frac{\lambda-\lambda_{h k l}}{2^{1 / 2} \sigma}+\frac{\sigma}{\tau}\right)\right],
\end{aligned}
$$

consisting of the solution of the integral of a Gaussian with deviation $\sigma$ convoluted with an exponential starting at $\lambda_{h k l}$ and decaying with a constant $\tau$. The edge parameters can be related to physical effects of the instrument and sample: $\sigma$ describes the broadening due to the sample and instrument geometry, $\tau$ describes the decay of the moderator (hence it is instrument dependent), and $\lambda_{h k l}$ is the Bragg-edge position. In contrast to the originally addressed transmission spectra (Santisteban et al., 2001), our data represent spectra of the actual attenuation coefficients, i.e. the total macroscopic cross section. Therefore, the analytical model has to be accordingly adapted. Correspondingly, the Kropff model $B$ was replaced by its complementary function, $1-B$, in order to achieve a step function equal to one on the lower-wavelength 
side of the edge and zero beyond the Bragg edge. The exponential functions describing the transmission before and after the edge were replaced by linear functions describing the attenuation before and after the edge. The fitting routine was written in Python using the Imfit package (Newville et al., 2014) and it can be found in the neutronimaging github repository (https://github.com/neutronimaging/ToFImaging).

\section{Results and discussions}

\subsection{White-beam tomographic reconstruction}

a size of $\sim 0.004 \mathrm{~mm}^{3}$. The total-cross-section spectra for isotropic powder-like materials are plotted as reference as dashed lines. Visual inspection confirms a relatively good agreement with the reference spectra in terms of overall spectral shape, single-edge position and height, especially for nickel, iron and titanium. In this respect, the worst match was found for aluminium and lead, as expected, these being the least attenuating and very low contrast materials. These observations were confirmed by computing the normalized cross-correlation (NCC) between the reference and measured values in the form of a similarity index. The NCC values are

Fig. 2 shows the resulting whitebeam tomography reconstruction: a slice taken at mid-height of the sample and a $3 \mathrm{D}$ rendering of the entire volume, with the Al matrix set to transparent and the different materials highlighted by false-color representation. The white-beam tomogram is of high quality and thus it can be used not only for the IAEA round-robin test but also to fine-tune all reconstruction parameters, because of the good statistics, low noise and good spatial resolution. The lower statistical quality of the individual wavelength tomograms is otherwise prone to introduce potential additional bias.

\subsection{Wavelength-resolved tomo- graphic reconstruction}

The wavelength-resolved tomography resulted in 165 volumes, each of $512 \times 512 \times 240$ voxels, corresponding to the 165 wavelength bins and a region of interest (ROI) of $512 \times 240$ detector pixels. Thus, this set of wavelengthdispersive tomographies maps in three dimensions the wavelength-dependent total cross section of each material in the sample. Fig. 3 shows 50 pixel vertical intensity line profiles centered on each material inset in the $\mathrm{Al}$ cylinder plotted against the wavelength. The observed vertical lines of changing contrast in the intensity patterns correspond to the Bragg edges of the respective materials.

In Fig. 4, the same information from the attenuation spectra is provided for $5 \times 5 \times 1$ voxel ROIs in the middle of each inset, and the corresponding median values are plotted against the wavelength. Given the pixel size of $0.055 \mathrm{~mm}$, this results in a macro voxel, corresponding to a gauge volume, with
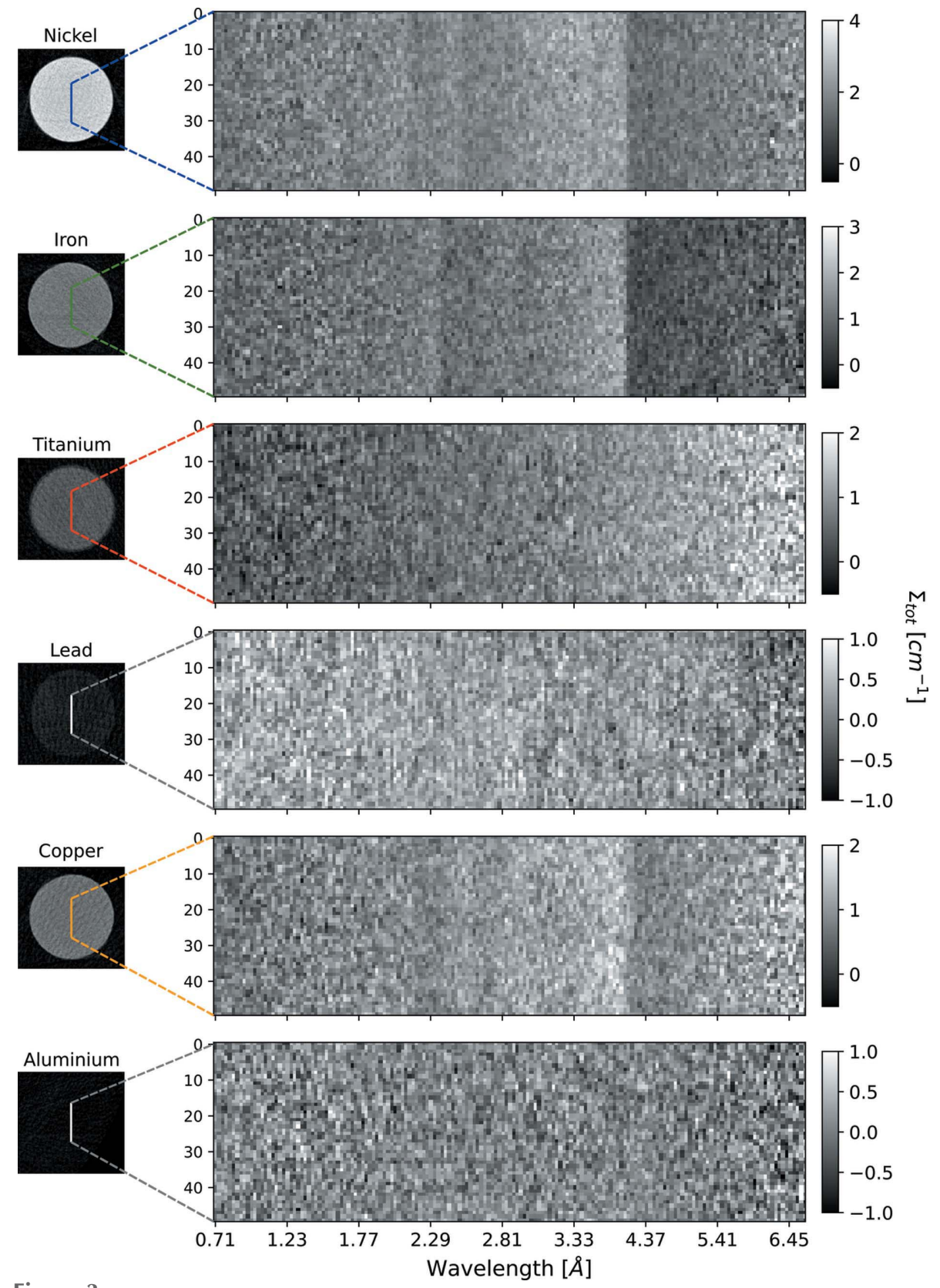

Figure 3

Wavelength-resolved slices. For each material, in a middle slice along the longitudinal axis of the sample, a 50 pixel line in the middle of each inset is shown for each reconstructed wavelength. Jumps in intensity contrast along the horizontal directions correspond to the Bragg-edge positions. On the left, custom ROIs show each inset and the line position on the white-beam reconstructed slice, for high-resolution visualization. 
provided in Fig. 4: very high similarity was found for nickel, iron, titanium and copper $(>0.7)$, while significantly lower values for lead (0.554) and especially aluminium (0.102) are obtained.

\subsection{Effect of background correction and deviation from reference spectra}

Both the wavelength-resolved and white-beam tomographic reconstructions were performed with and without background
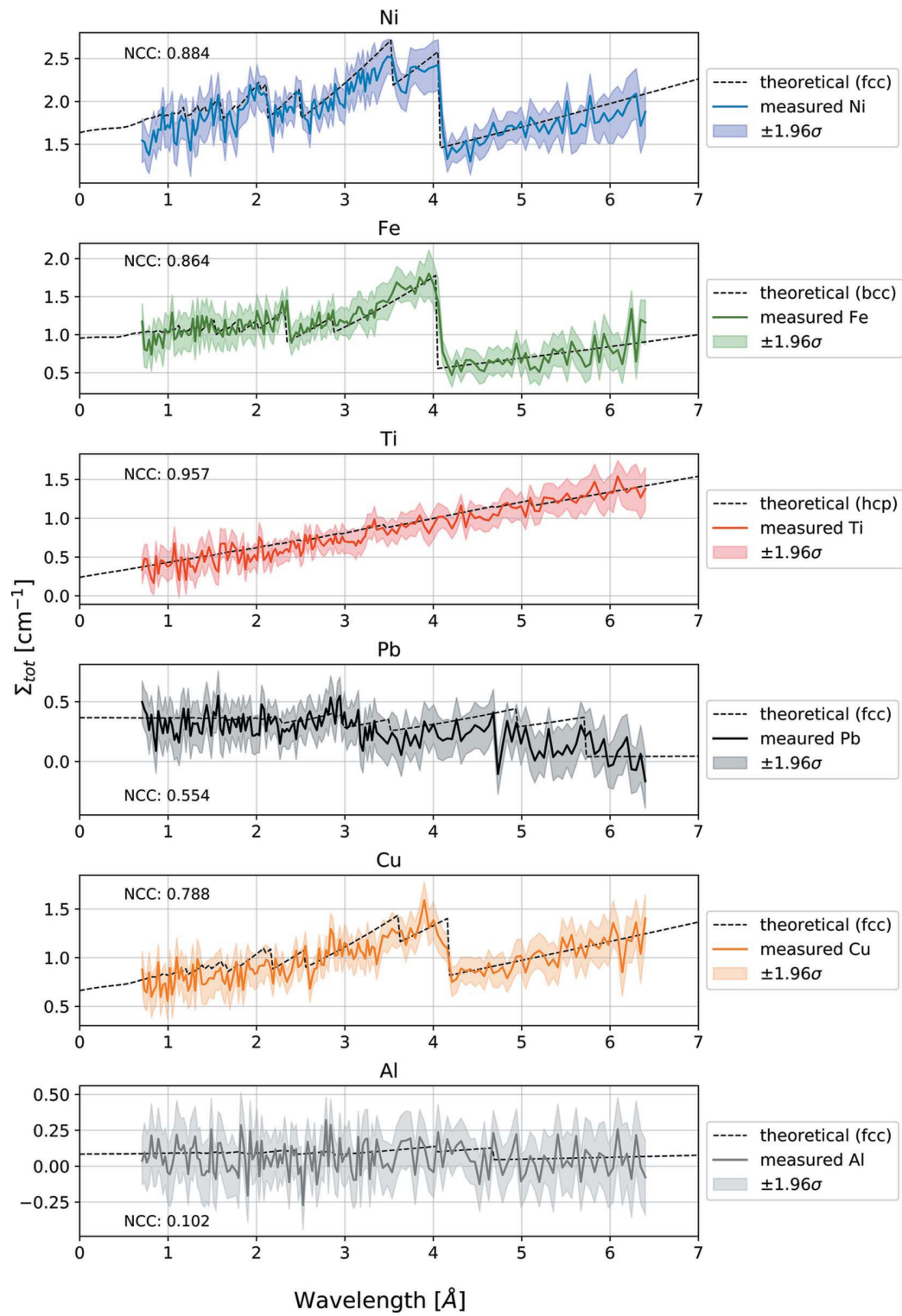

Figure 4

Full spectrum computation from the wavelength-resolved tomographic reconstructions. In a mid-height slice along the longitudinal axis of the sample, an ROI of $5 \times 5 \times 1$ voxels was chosen in the center of the insets. The median value of this ROI is plotted against the wavelength with a colored line. The error bars are shown in partially transparent colors and were computed as the \pm 1.96 standard deviation of the binned images $(5 \times 5 \times 1$ voxel ROIs). The dashed lines are the total cross sections for the ideal isotropic powder-like materials computed with the nxsplotter software. The normalized cross-correlation is computed between the reference signals and those calculated from measurement as an indicator of similarity. [fcc: face-centered cubic; bcc: body-centered cubic; hcp: hexagonal close packed.] correction to evaluate the effect and optimize the background stimate. Instrument-related effects and sample scattering are detector at the time of the experiment, we did not have an detector background effects. However, in a measurement detector, we have quantitatively measured the level of background with the black body method (Boillat et al., 2018; Carminati et al., 2019), and we observed values of additive background of the order of magnitude of $5 \%$ of the open-beam image. This gave us a further indication that background correction is also necessary to obtain quantitative results in such a configuration. We therefore investigated the effect of correction of our data by subtracting an empirically estimated constant background, defined for each pixel as a fraction of the measured signal. Background values equal to 5,7 and $10 \%$ of the median value of the sample image for each wavelength were considered.

Fig. 5 shows the effect of background correction on the attenuation spectra from the wavelength-resolved tomography. Here, we plot the spectra obtained by averaging largervoxel ROIs for a better visualization $(50 \times$ $50 \times 1$ voxels). Without background correction, systematic underestimation of the total cross section is visible for all wavelengths. In all cases, optimal results were found when correcting with an estimated background of $7 \%$, supported in particular by the very good matching with reference spectra at the absorption tail of the spectra, i.e. in the region where Bragg scattering is no longer taking place. Results obtained with 5 and $10 \%$ are shown in the supplementary material (Fig. S1). It is noticeable that the $5 \%$ level resulted in underestimation of the additive background, while $10 \%$ best matched the absorption tails but overestimated, especially for $\mathrm{Ni}, \mathrm{Fe}$ and $\mathrm{Cu}$, the region of the main Bragg edges, where forward scattering is expected to be less prominent.

For the white-beam tomography, the expected linear attenuation coefficients were obtained from the instrument and the computed wavelength-dependent cross section for each material. Fig. 6 shows the normalized (to maximum) spectrum of IMAT measured with the MCP detector as well as the reference total cross sections of the six materials computed with the nxsplotter software (Boin, 
2012). From these data, the expected linear attenuation coefficient for the white-beam tomography can be obtained by calculating the weighted mean of the attenuation coefficients with respect to the reference neutron spectrum: $\mu=$ $\int \phi(\lambda) \Sigma(\lambda) \mathrm{d} \lambda / \int \phi(\lambda) \mathrm{d} \lambda$, where $\Sigma(\lambda)$ is the total cross section (Fig. 6 lower panel) and $\phi(\lambda)$ is the measured spectrum as a function of the wavelength $\lambda$, thus including the neutron capture efficiency (Fig. 6 upper panel).

The attenuation coefficients calculated from the weighted reference cross section and those calculated from measurements without correction and with the different empirical background corrections are shown in Fig. 7. The calculated linear attenuation coefficients were computed from a midheight slice by selecting square ROIs of $50 \times 50$ pixels centered on each specific material inset. As expected, the linear attenuation coefficients were systematically underestimated without background correction and clearly improve when the correction is applied. Results that best match reference expectations are obtained when correcting for a background estimate equal to $10 \%$ of the sample image. However, this would be valid if the measured spectra exactly matched the reference ones. For the studied sample, as shown in Figs. 4 and 5, deviations from the reference spectra are noticeable. These deviations are due to the presence of texture and impurities, very common for conventional engineering materials like those contained in the studied object. A typical example is copper, where electron backscatter diffraction investigations revealed a strong $\langle 111\rangle$ crystallographic texture along the direction of the longitudinal axis of the cylindrical sample, due to the thermomechanical production process, as shown in Figure S2 of the supplementary materials. The absence of $\{111\}$ planes along the radial direction of the cylinder, which is the transmitted beam direction of the tomography setup, results in a weak or almost absent (111) Bragg edge for $\mathrm{Cu}$ as seen in Fig. 4. Therefore, the attenuation coefficients for the white-beam tomography could be expected
Without background correction
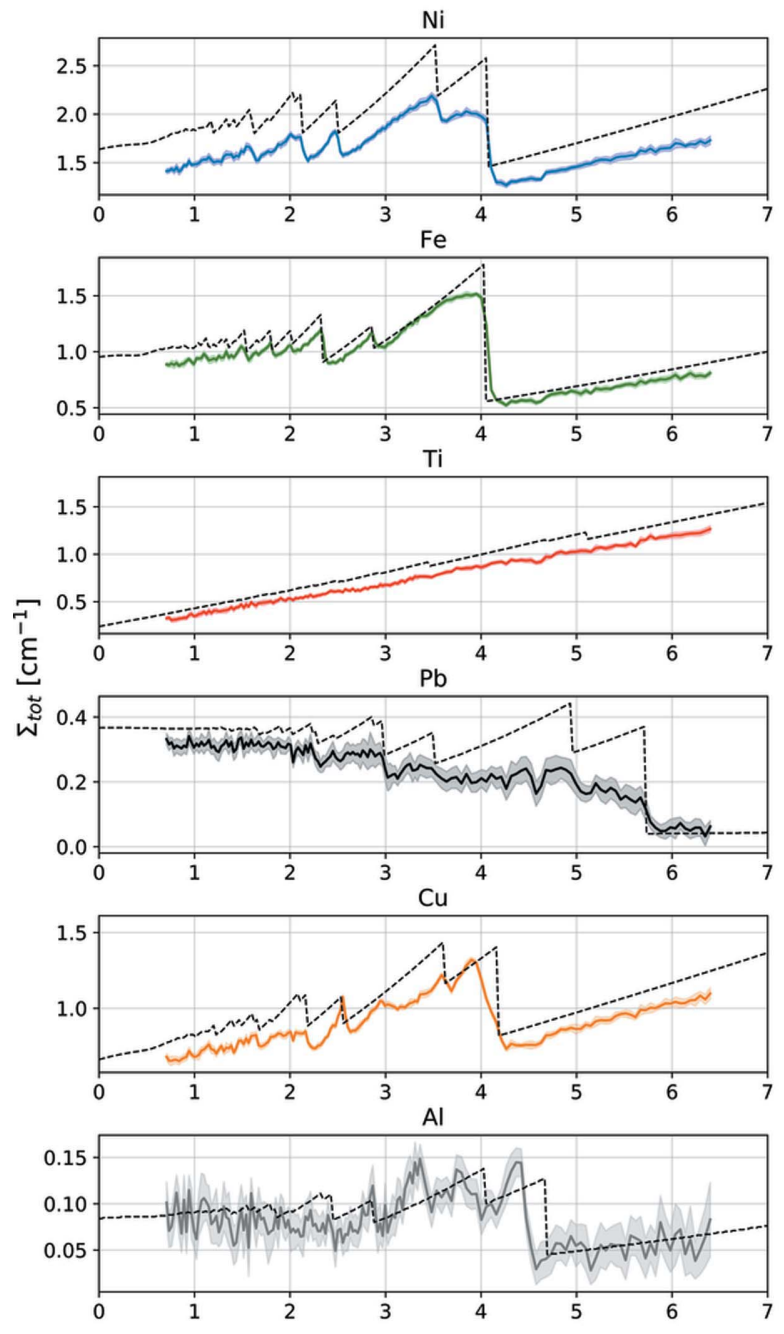

With background correction
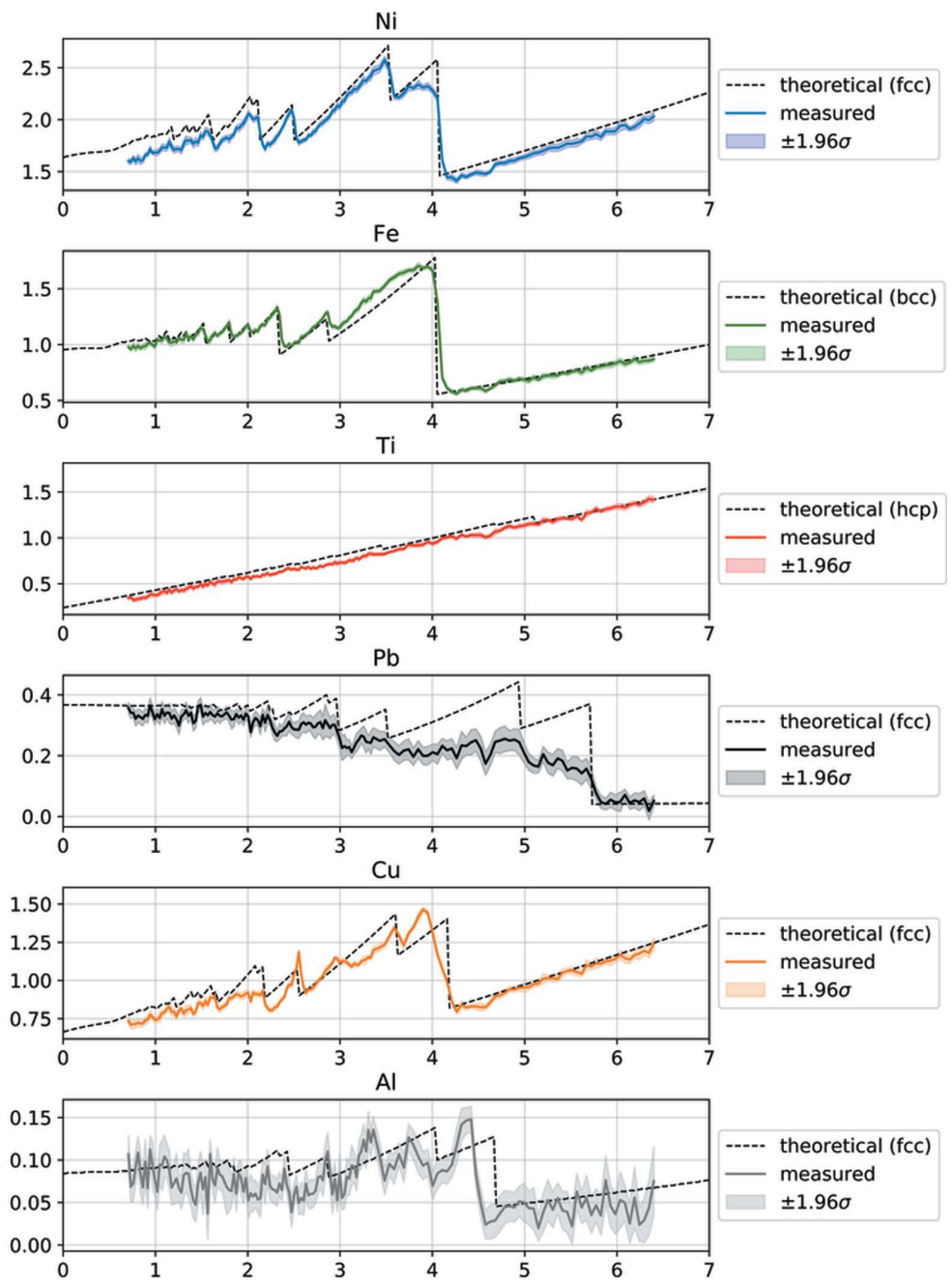

Figure 5

Wavelength $[\AA]$

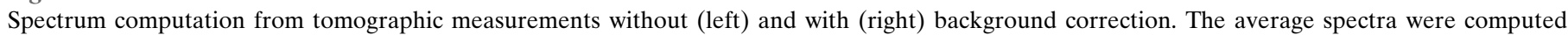

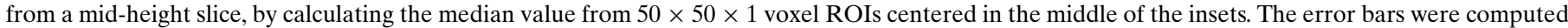
as \pm 1.96 standard deviation of the $50 \times 50 \times 1$ voxel ROIs along the sample. 
to be closer to those obtained when convolving the measured spectra obtained with $7 \%$ wavelength-dependent correction with the instrument spectrum (pink bar in Fig. 7). By taking this value as reference, an overall $10 \%$ correction seems to
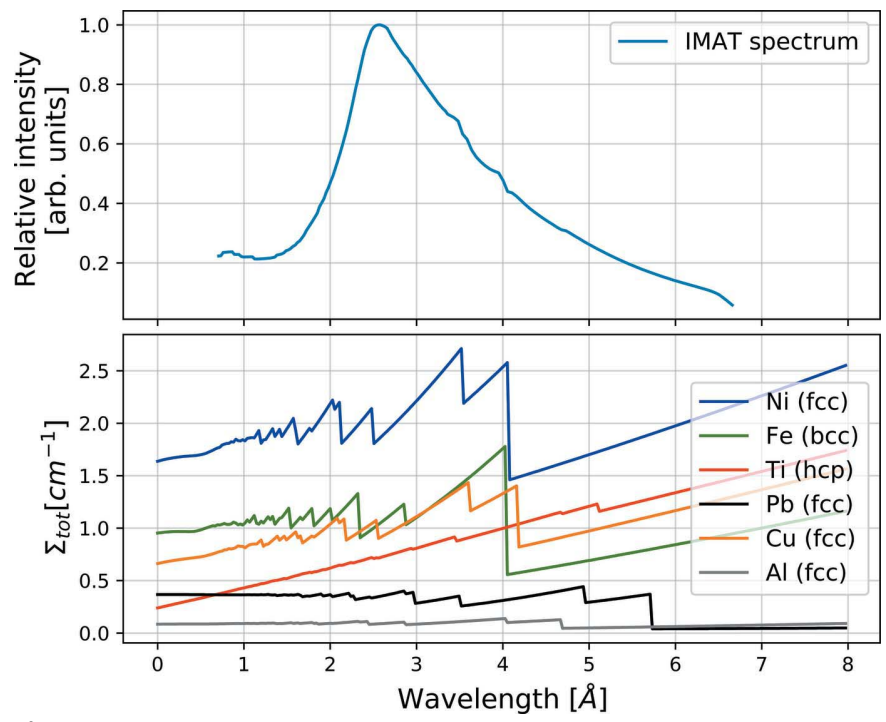

Figure 6

Top: measured wavelength spectrum of IMAT. Bottom: reference total cross section of the studied materials computed with nxsPlotter (Boin, 2012).

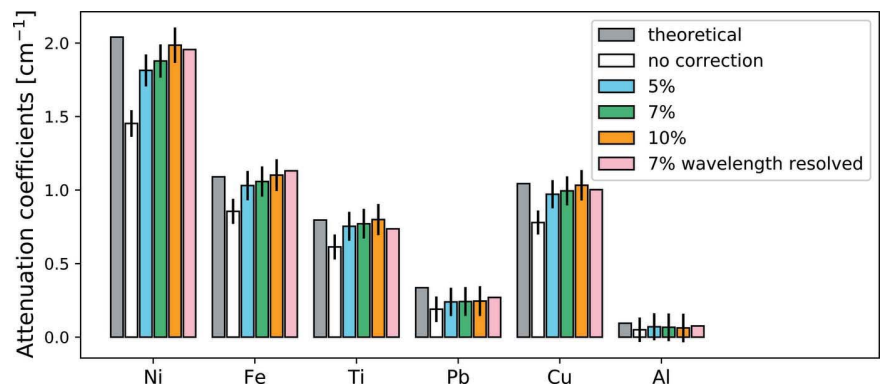

Figure 7

Reference and experimental linear attenuation coefficients, obtained with different background corrections, where the constant background was set to 5,7 and $10 \%$ of the open beam. The pink bar indicates the attenuation coefficients computed from the spectra obtained when correcting for $7 \%$ of the image signal.
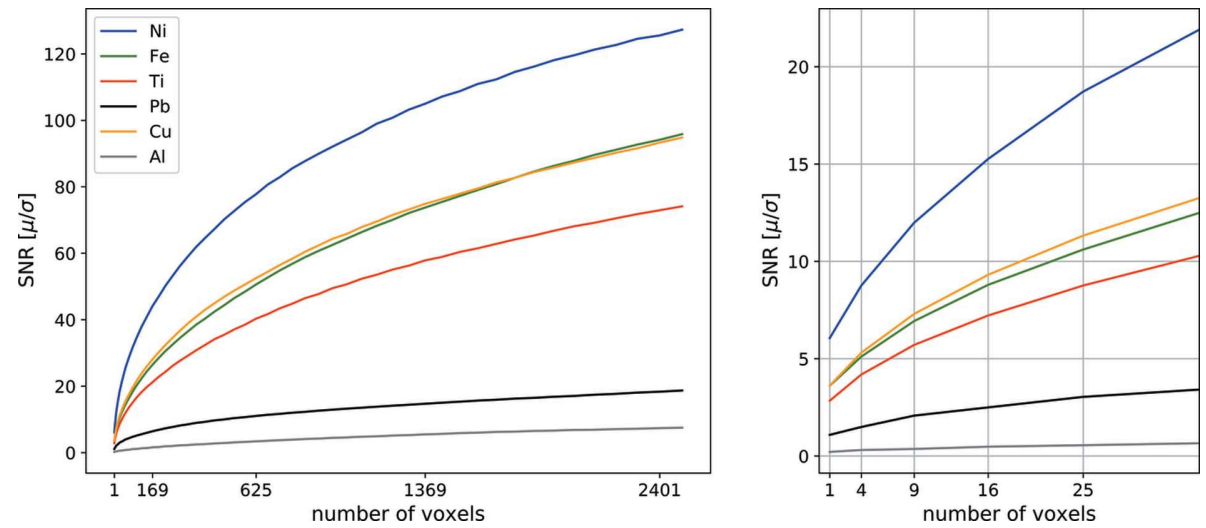

Figure 8

Signal-to-noise ratio of the tomographic volumes against the number of voxels used as ROI. On the right, a zoomed region is shown for all materials to allow the visualization for the smaller bin size. overestimate the attenuation coefficients for white-beam tomography, in particular for nickel, titanium and copper, as also noted in the wavelength-resolved tomography study.

In general, the correction with a background fraction that is constant throughout the field of view seemed to be a good first approximation in the presented case and it compensated well the apparent systematic bias. The improvement is less significant or not even noticeable for lead and aluminium, where, being transparent to neutrons, the values of their attenuation coefficients are close to or smaller than the achieved statistical error and hence their overall variation.

\subsection{Smallest gauge volume for Bragg-edge fitting and analysis}

In order to investigate the smallest gauge volume that allows one to retrieve reliable local-cross-section spectra, we have investigated how the SNR and the contrast-to-noise ratio (CNR) relate to the number of voxels in the reconstructed tomographies binned into an ROI. For this study, we considered a subset of the total tomographic volume made of 100 slices, centered at mid-height along the sample longitudinal axis. From those, square cross-sectional ROIs (height fixed at 1 voxel) were used to bin the tomographic volume with an increasing number of voxels, ranging from $1 \times 1 \times 1$ to $50 \times$ $50 \times 1$ voxels, translating into physical volumes from $0.055 \times$ $0.055 \times 0.055 \mathrm{~mm}$ to $2.75 \times 2.75 \times 0.055 \mathrm{~mm}$. For each inset, the wavelength-resolved SNR was defined as the mean value divided by the standard deviation of the binned images for each wavelength, and then averaged over the wavelength range. In order to have a metric related to the Bragg-edge features, the CNR was defined as the ratio between the nominal edge height and the standard deviation of the binned images averaged along the wavelength axis, for the different bin sizes.

Computed SNRs for each material are plotted in Fig. 8. As expected in the case of statistical noise, the SNR increases with increasing number of voxels used for binning, with higher SNR values, in decreasing order, for nickel, iron, copper and titanium, and significantly lower values for lead and aluminium. The highest SNR resulted for $\mathrm{Ni}$, with values above 10 when considering a bin size of $3 \times 3 \times 1$. Iron and copper showed very similar values to each other, with values of around 7 for $3 \times$ $3 \times 1$ bin size.

For the CNR evaluation, we have considered the main Bragg edge for nickel (111), iron (110) and copper (111), as those materials exhibit the most prominent Bragg edges which were also clearly visible in the measured spectra. The edge heights from the reference spectra were computed as the maximum intensity minus the minimum intensity before and after the edge and resulted in 1.221, 1.11 and $0.58 \AA$, respectively, for $\mathrm{Ni}, \mathrm{Fe}$ and $\mathrm{Cu}$. Results of the 
Table 1

Edge positions for isotropic powder-like materials (reference) and computed from the data (fitted).

The errors were estimated as the standard deviation of the $\lambda_{h k l}$ parameter in the fitting model, computed as the square root of the corresponding diagonal element of the covariance matrix and obtained when fitting only the edge position parameter and keeping the others fixed.

\begin{tabular}{lll}
\hline Sample $(h k l)$ & Reference $(\AA)$ & Fitted $(\AA)$ \\
\hline $\mathrm{Ni}(111)$ & 4.07 & $4.090 \pm 0.007$ \\
$\mathrm{Ni}(200)$ & 3.542 & $3.582 \pm 0.012$ \\
$\mathrm{Ni}(220)$ & 2.492 & $2.517 \pm 0.010$ \\
$\mathrm{Ni}(311)$ & 2.126 & $2.139 \pm 0.007$ \\
& & \\
Fe (110) & 4.054 & $4.072 \pm 0.003$ \\
$\mathrm{Fe}(200)$ & 2.866 & $2.883 \pm 0.022$ \\
Fe (211) & 2.34 & $2.369 \pm 0.011$ \\
\hline
\end{tabular}
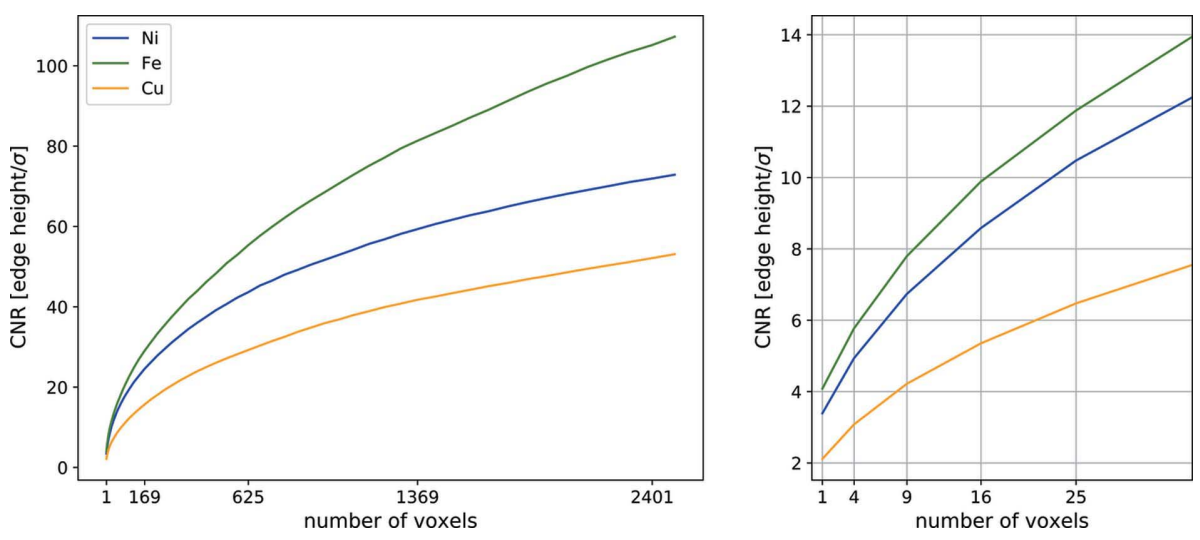

Figure 9

Contrast-to-noise ratio of the tomographic volumes against the number of voxels used as ROI calculated for the main Bragg edge of nickel, iron and copper. On the right, a zoomed region is shown for all three materials to allow the visualization for the smaller bin size.
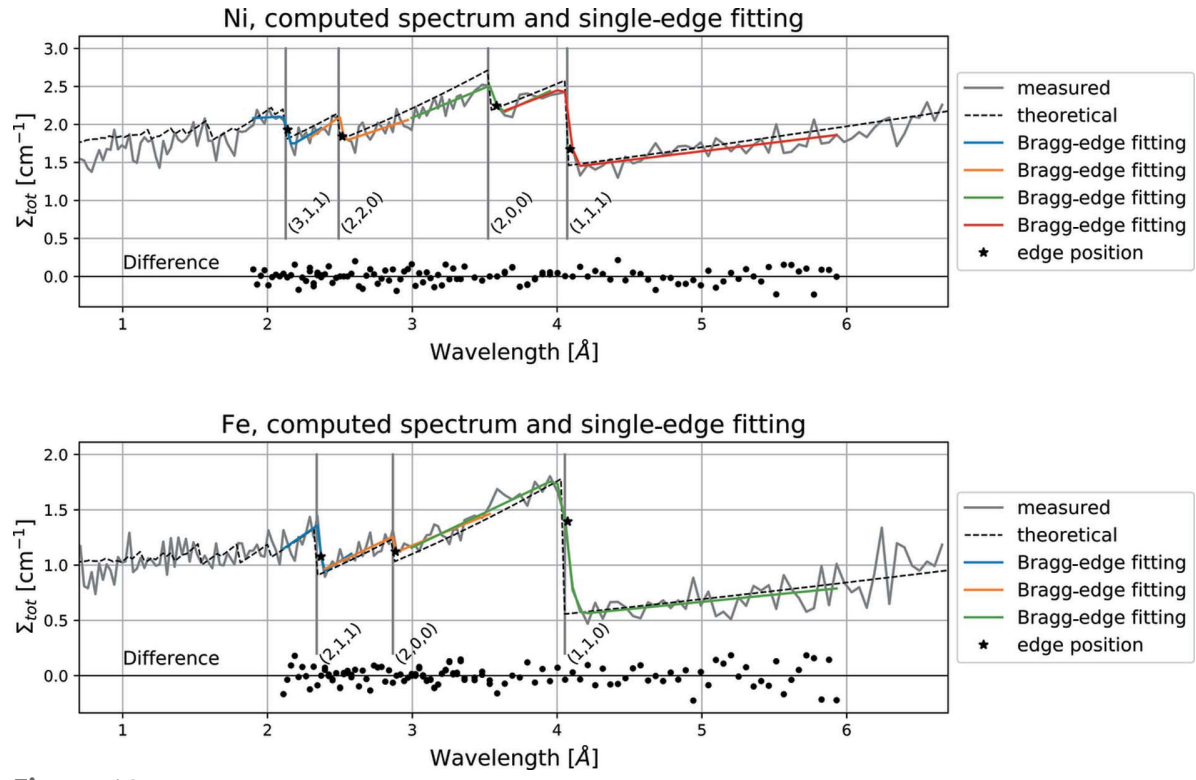

Figure 10

Single-Bragg-edge fitting on attenuation spectra computed from a $5 \times 5 \times 1$ voxel ROI for nickel and iron. Different colors for each edge fitting were chosen for better visualization. The star-shaped markers plot the edge position as computed from the Bragg-edge model. The point-by-point difference between fitted and measured data is shown at the bottom of each plot. The continuous line represents the mean difference. computed CNR are shown in Fig. 9. The CNR highlights that the best contrast is achievable for iron and, compared with SNR, better differentiates between the three materials.

As example cases for Bragg-edge fitting, we have therefore considered the spectra presented in Fig. 4 (obtained with a $5 \times$ $5 \times 1$ voxel ROI) for the nickel and iron samples, as these are the materials characterized by more pronounced Bragg edges. For this bin size, both CNR and SNR are above 10 for both iron and copper. Results on single-edge fitting are shown in Fig. 10: the routine was able to fit efficiently the four and three most prominent Bragg edges, for nickel and iron, respectively, with a mean difference between the fit and the fitted data close zero for all cases.

The Bragg-edge positions resulted from fitting with the Bragg-edge model (described in Section 3.3) and are marked in Fig. 10. Table 1 shows the computed Bragg-edge positions for specific lattice plane families $(h k l)$ for iron and nickel, and those obtained for isotropic powder-like materials from the Bragg law: $\lambda=2 d_{h k l}=$ $2 a\left(h^{2}+k^{2}+l^{2}\right)^{-1 / 2}, a$ being the lattice constant for each material. From the fitted edge positions, the lattice constants are measured to be $3.558 \pm 0.015$ and $2.889 \pm 0.009 \AA$ for nickel (averaged from the computed $d_{111}, d_{200}, d_{220}$ and $d_{311}$ ) and iron (averaged from the computed $d_{110}, d_{220}$ and $d_{211}$ ), respectively.

\section{Conclusions}

We have demonstrated the feasibility of a 4D wavelength-resolved TOF tomography study of a polycrystalline sample containing multiple phases. Our results underline the potential of a TOF imaging beamline such as IMAT to measure and reconstruct well resolved wavelength-dependent attenuation spectra at the voxel level. Therefore, mapping of the wavelength-dependent cross section containing local crystallographic information becomes possible for isotropic fine-grained polycrystalline samples.

It was shown that background correction plays an essential role and has to be considered carefully in order to obtain results on an absolute scale. In future research, we foresee quantitatively measuring the background wavelength dispersively using the black-body method (Boillat et al., 2018; Carminati et al., 2019), to also carefully compensate for wavelength-dependent sample scattering close to the forward direction, 
which varies significantly depending on the sample geometry and composition.

Quantitative Bragg-edge fitting can be performed on small gauge volumes and holds potential for local crystallographic characterization such as extraction of lattice constants or Bragg-edge parameters related, for example, to the phase fractions. Here the corresponding spatial resolution was of the order of $275 \mu \mathrm{m}$ (from a $5 \times 5 \times 1 \mathrm{ROI}$, given $55 \mu \mathrm{m}$ pixel size), depending on data quality. We therefore assume the specific sample to also be an excellent round-robin test sample for TOF beamlines and tomography, though it might need to be complemented by other references such as, for example, those used for strain mapping.

\section{References}

Abbey, B., Zhang, S. Y., Vorster, W. \& Korsunsky, A. M. (2009). Proc. Eng. 1, 185-188.

Boillat, P., Carminati, C., Schmid, F., Grünzweig, C., Hovind, J., Kaestner, A., Mannes, D., Morgano, M., Siegwart, M., Trtik, P., Vontobel, P. \& Lehmann, E. (2018). Opt. Express, 26, 15769-15784. Boin, M. (2012). J. Appl. Cryst. 45, 603-607.

Brooks, A. J., Yao, H., Yuan, J., Kio, O., Lowery, C. G., Markötter, H., Kardjilov, N., Guo, S. \& Butler, L. G. (2018). Additive Manufacturing, 22, 658-664.

Carminati, C., Boillat, P., Schmid, F., Vontobel, P., Hovind, J., Morgano, M., Raventos, M., Siegwart, M., Mannes, D., Gruenzweig, C., Trtik, P., Lehmann, E., Strobl, M. \& Kaestner, A. (2019). PLoS One, 14, e0210300.

Hendriks, J. N., Gregg, A. W. T., Wensrich, C. M., Tremsin, A. S., Shinohara, T., Meylan, M., Kisi, E. H., Luzin, V. \& Kirsten, O. (2017). Phys. Rev. Mater. 1, 053802.

Iwase, K., Sato, H., Harjo, S., Kamiyama, T., Ito, T., Takata, S., Aizawa, K. \& Kiyanagi, Y. (2012). J. Appl. Cryst. 45, 113-118.

Kaestner, A. P. (2011). Nucl. Instrum. Methods Phys. Res. A, 651, 156-160.

Kaestner, A. \& Carminati, C. (2019). neutronimaging/imagingsuite: Improved Processing Accuracy and Added UI Features, https:// doi.org/10.5281/zenodo.3229672.

Kaestner, A., Hartmann, S., Kühne, G., Frei, G., Grünzweig, C., Josic, L., Schmid, F. \& Lehmann, E. (2011). Nucl. Instrum. Methods Phys. Res. A, 659, 387-393.

Kaestner, A., Lehmann, E., Hovind, J., Radebe, M., de Beer, F. \& Sim, C. (2013). Phys. Proc. 43, 128-137.

Kardjilov, N., Baechler, S., Bastürk, M., Dierick, M., Jolie, J., Lehmann, E., Materna, T., Schillinger, B. \& Vontobel, P. (2003). Nucl. Instrum. Methods Phys. Res. A, 501, 536-546.

Kardjilov, N., Schillinger, B. \& Steichele, E. (2004). Appl. Radiat. Isot. 61, 455-460.

Kockelmann, W., Frei, G., Lehmann, E., Vontobel, P. \& Santisteban, J. (2007). Nucl. Instrum. Methods Phys. Res. A, 578, 421-434.

Kropff, F., Granada, J. R. \& Mayer, R. E. (1982). Nucl. Instrum. Methods Phys. Res. 198, 515-521.

Lehmann, E., Frei, G., Vontobel, P., Josic, L., Kardjilov, N., Hilger, A., Kockelmann, W. \& Steuwer, A. (2009). Nucl. Instrum. Methods Phys. Res. A, 603, 429-438.

Lehmann, E. H., Vontobel, P. \& Wiezel, L. (2001). Nondestr. Test. Eval. 16, 191-202.

Makowska, M. G., Kuhn, L. T., Frandsen, H. L., Lauridsen, E. M., De Angelis, S., Cleemann, L. N., Morgano, M., Trtik, P. \& Strobl, M. (2017). J. Power Sources, 340, 167-175.

Makowska, M. G., Strobl, M., Lauridsen, E. M., Frandsen, H. L., Tremsin, A. S., Kardjilov, N., Manke, I., Kelleher, J. F. \& Theil Kuhn, L. (2015). J. Appl. Cryst. 48, 401-408.

Makowska, M. G., Strobl, M., Lauridsen, E. M., Kabra, S., Kockelmann, W., Tremsin, A., Frandsen, H. L. \& Theil Kuhn, L. (2016). J. Appl. Cryst. 49, 1674-1681.
Minniti, T. (2019). J. Appl. Cryst. 52, 903-909.

Minniti, T., Watanabe, K., Burca, G., Pooley, D. E. \& Kockelmann, W. (2018). Nucl. Instrum. Methods Phys. Res. A, 888, 184-195.

Newville, M., Stensitzki, T., Allen, D. B. \& Ingargiola, A. (2014). Lmfit: Non-linear Least-Square Minimization and Curve-Fitting for Python, https://zenodo.org/record/11813.

Ramadhan, R. S., Kockelmann, W., Minniti, T., Chen, B., Parfitt, D., Fitzpatrick, M. E. \& Tremsin, A. S. (2019). J. Appl. Cryst. 52, 351368.

Raventós, M., Lehmann, E. H., Boin, M., Morgano, M., Hovind, J., Harti, R., Valsecchi, J., Kaestner, A., Carminati, C., Boillat, P., Trtik, P., Schmid, F., Siegwart, M., Mannes, D., Strobl, M. \& Grünzweig, C. (2018). J. Appl. Cryst. 51, 386-394.

Santisteban, J., Edwards, L., Fizpatrick, M., Steuwer, A. \& Withers, P. (2002a). Appl. Phys. Mater. Sci. Process. 74, s1433-s1436.

Santisteban, J., Edwards, L., Fitzpatrick, M., Steuwer, A., Withers, P., Daymond, M., Johnson, M., Rhodes, N. \& Schooneveld, E. (2002b). Nucl. Instrum. Methods Phys. Res. A, 481, 765-768.

Santisteban, J. R., Edwards, L. \& Stelmukh, V. (2006). Physica B, 385386, 636-638.

Santisteban, J. R., Edwards, L., Steuwer, A. \& Withers, P. J. (2001). J. Appl. Cryst. 34, 289-297.

Santisteban, J., Vicente-Alvarez, M., Vizcaino, P., Banchik, A., Vogel, S., Tremsin, A., Vallerga, J., McPhate, J., Lehmann, E. \& Kockelmann, W. (2012). J. Nucl. Mater. 425, 218-227.

Sato, H. (2017). J. Imaging, 4, 7.

Song, G., Lin, J. Y. Y., Bilheux, J. C., Xie, Q., Santodonato, L. J., Molaison, J. J., Skorpenske, H. D. M., Dos Santos, A., Tulk, C. A., An, K., Stoica, A. D., Kirka, M. M., Dehoff, R. R., Tremsin, A. S., Bunn, J., Sochalski-Kolbus, L. M. \& Bilheux, H. Z. (2017). J. Imaging, 3, 65.

Steuwer, A., Santisteban, J., Withers, P. \& Edwards, L. (2004). Physica $B$, 350, 159-161.

Steuwer, A., Santisteban, J. R., Withers, P. J., Edwards, L. \& Fitzpatrick, M. E. (2003). J. Appl. Cryst. 36, 1159-1168.

Steuwer, A., Withers, P. J., Santisteban, J. R. \& Edwards, L. (2005). J. Appl. Phys. 97, 074903.

Steuwer, A., Withers, P., Santisteban, J., Edwards, L., Bruno, G., Fitzpatrick, M., Daymond, M., Johnson, M. \& Wang, D. (2001). Phys. Status Solidi A, 185, 221-230.

Strobl, M., Woracek, R., Kardjilov, N., Hilger, A., Wimpory, R., Tremsin, A., Wilpert, T., Schulz, C., Manke, I. \& Penumadu, D. (2012). Nucl. Instrum. Methods Phys. Res. A, 680, 27-34.

Treimer, W., Strobl, M., Kardjilov, N., Hilger, A. \& Manke, I. (2006). Appl. Phys. Lett. 89, 203504.

Tremsin, A. S., Vallerga, J. V., McPhate, J. B. \& Siegmund, O. H. W. (2014). J. Instrum. 9, C05026.

Tremsin, A., Vallerga, J., McPhate, J. \& Siegmund, O. (2015). Nucl. Instrum. Methods Phys. Res. A, 787, 20-25.

Venkatakrishnan, S. V., Dessieux, L. \& Bingham, P. (2019). 2019 IEEE International Conference on Acoustics, Speech, and Signal Processing Proceedings, pp. 7700-7704, https://doi.org/10.1109/ icassp.2019.8683013. The Institute of Electrical and Electronics Engineers.

Vitucci, G., Minniti, T., Di Martino, D., Musa, M., Gori, L., Micieli, D., Kockelmann, W., Watanabe, K., Tremsin, A. \& Gorini, G. (2018). Microchem. J. 137, 473-479.

Wensrich, C., Hendriks, J., Gregg, A., Meylan, M., Luzin, V. \& Tremsin, A. (2016a). Nucl. Instrum. Methods Phys. Res. B, 383, 52-58.

Wensrich, C. M., Hendriks, J. N. \& Meylan, M. H. (2016b). Strain, 52, $80-87$.

Woracek, R., Penumadu, D., Kardjilov, N., Hilger, A., Boin, M., Banhart, J. \& Manke, I. (2014). Adv. Mater. 26, 4069-4073.

Woracek, R., Penumadu, D., Kardjilov, N., Hilger, A., Strobl, M., Wimpory, R. C., Manke, I. \& Banhart, J. (2011). J. Appl. Phys. 109, 093506.

Woracek, R., Santisteban, J., Fedrigo, A. \& Strobl, M. (2018). Nucl. Instrum. Methods Phys. Res. A, 878, 141-158. 